\title{
Convergence of a Generalized USOR Iterative Method for Augmented Systems
}

\author{
Yu-Qin Bai, ${ }^{1,2}$ Ting-Zhu Huang, ${ }^{1}$ and Miao-Miao $\mathbf{Y u}^{3}$ \\ ${ }^{1}$ School of Mathematical Sciences, University of Electronic Science and Technology of China, Chengdu, Sichuan 611731, China \\ ${ }^{2}$ School of Computer Science and Information Engineering, Northwest University for Nationalities, Lanzhou, Gansu 730030, China \\ ${ }^{3}$ School of Science, Sichuan University of Science and Engineering, Zigong, Sichuan 643000, China
}

Correspondence should be addressed to Yu-Qin Bai; yqbai2006@163.com

Received 3 June 2013; Accepted 18 September 2013

Academic Editor: Manuel Ruiz Galan

Copyright (C) 2013 Yu-Qin Bai et al. This is an open access article distributed under the Creative Commons Attribution License, which permits unrestricted use, distribution, and reproduction in any medium, provided the original work is properly cited.

\begin{abstract}
Zhang and Shang (2010) have presented the Uzawa-SOR (USOR) algorithm to solve augmented systems. In this paper, we establish a generalized Uzawa-SOR (GUSOR) method for solving augmented systems, which is the extension of the USOR method. We prove the convergence of the proposed method under suitable restrictions on the iteration parameters. Lastly, numerical experiments are carried out and experimental results show that our proposed method with appropriate parameters has faster convergence rate than the USOR method.
\end{abstract}

\section{Introduction}

We consider the solution of systems of linear equations of the 2-by-2 block structure as follows:

$$
\left(\begin{array}{cc}
A & B \\
B^{T} & 0
\end{array}\right)=\left(\begin{array}{l}
b \\
q
\end{array}\right),
$$

where $A \in R^{m \times m}$ is a symmetric and positive definite matrix and $B \in R^{m \times n}$ is a matrix of full column rank, $x, p \in R^{m}$, $y, q \in R^{n}$, and $m \geq n$. $B^{T}$ denotes the transpose of the matrix $B$. We assume that they are of the appropriate dimensions whenever the zero matrix $O$ and the identity matrix $I$ are used in this paper. The linear systems (1) appears in many different applications of scientific computing, such as the mixed finite element for incompressible flow problems when some form of pressure stabilization is included in the discretization, constrained optimization [1], computational fluid dynamics, and Stokes problems, of constrained least squares problems, and generalized least squares problems $[2-5]$; see $[6,7]$ and references therein.

A large variety of methods for solving linear systems of the form (1) can be found in the literature. Yuan and Iusem [3, 5] presented variants of the SOR method and preconditioned conjugate gradient methods. Golub et al. [8] proposed SORlike algorithms for solving the augmented systems, which was further accelerated and generalized by GSOR method in [9]. Darvishi and Hessari [10] studied the SSOR method. Zhang and Lu [11] studied a GSSOR (generalized SSOR) method. Recently, Zhang and Shang [12] proposed the Uzawa-SOR method and studied its convergence. Bai and Wang [13] established and studied the parameterized inexact Uzawa (PIU) method for solving the corresponding saddle point problems, which was also discussed convergence conditions for matrix splitting iteration methods in [14].

The remainder of the paper is organized as follows. In Section 2, we establish a generalized Uzawa-SOR (GUSOR) method for solving augmented systems and analyze convergence of the corresponding method in Section 3. Numerical results are presented in Section 4. At last, we give some remarks in Section 5.

\section{Generalized Uzawa-SOR (GUSOR) Method}

For the sake of simplicity, we rewrite system (1) as

$$
\left(\begin{array}{cc}
A & B \\
-B^{T} & 0
\end{array}\right)=\left(\begin{array}{c}
b \\
-q
\end{array}\right)
$$


where $A \in R^{m \times m}$ is a symmetric and positive definite matrix and $B \in R^{m \times n}$ is a matrix of full column rank. Let $A$ be decomposed as $A=D-L-U$ in which $D$ is the diagonal of $A, L$ is the strict lower part of $A$, and $U$ is the strict upper part of $A$ with $\omega$ and $\tau$ being nonzero reals.

To construct the generalized USOR method, we consider the following splitting:

$$
\begin{aligned}
\mathscr{A} & =\left(\begin{array}{cc}
A & B \\
-B^{T} & 0
\end{array}\right)=\left(\begin{array}{cc}
D-L-U & B \\
-B^{T} & 0
\end{array}\right) \\
& =\mathscr{D}-\mathscr{L}-\mathscr{U},
\end{aligned}
$$

where

$$
\begin{gathered}
\mathscr{D}=\left(\begin{array}{ll}
D & O \\
O & Q
\end{array}\right), \quad \mathscr{L}=\left(\begin{array}{cc}
L & O \\
B^{T} & \alpha Q
\end{array}\right), \\
\mathscr{U}=\left(\begin{array}{cc}
U & -B \\
O & \beta Q
\end{array}\right),
\end{gathered}
$$

$Q \in \mathbb{R}^{n \times n}$ is a prescribed a symmetric positive definite matrix and $\alpha+\beta=1$. Let $\omega$ and $\tau$ be two nonzero reals, let $I_{m} \in$ $R^{m \times m}$ and $I_{n} \in R^{n \times n}$ be the $m$-by- $m$ and the $n$-by- $n$ identity matrices, respectively, and let $\Omega$ be given parameter matrices of the form

$$
\Omega=\left(\begin{array}{cc}
\omega I_{m} & O \\
O & \tau I_{n}
\end{array}\right) .
$$

Then we consider the following generalized SOR iteration scheme for solving the augmented linear system (2):

$$
\begin{aligned}
\left(\begin{array}{c}
x^{(k+1)} \\
y^{(k+1)}
\end{array}\right)= & (\mathscr{D}-\Omega \mathscr{L})^{-1}[(I-\Omega) \mathscr{D}+\Omega \mathscr{U}] \\
& \times\left(\begin{array}{c}
x^{(k)} \\
y^{(k)}
\end{array}\right)+(\mathscr{D}-\Omega \mathscr{L})^{-1} \Omega\left(\begin{array}{c}
b \\
-q
\end{array}\right),
\end{aligned}
$$

or equivalently,

$$
\begin{aligned}
\left(\begin{array}{l}
x^{(k+1)} \\
y^{(k+1)}
\end{array}\right)= & H(\omega, \tau, \alpha)\left(\begin{array}{l}
x^{(k)} \\
y^{(k)}
\end{array}\right) \\
& +M(\omega, \tau, \alpha)^{-1}\left(\begin{array}{c}
b \\
-q
\end{array}\right),
\end{aligned}
$$

where

$$
\begin{aligned}
H(\omega, \tau, \alpha) \equiv & (\mathscr{D}-\Omega \mathscr{L})^{-1}[(I-\Omega) \mathscr{D}+\Omega \mathscr{U}] \\
= & \left(\begin{array}{cc}
(D-\omega L)^{-1} & O \\
\frac{\tau}{1-\alpha \tau} Q^{-1} B^{T}(D-\omega L)^{-1} & \frac{1}{1-\alpha \tau} Q^{-1}
\end{array}\right)\left(\begin{array}{cc}
(1-\omega) D+\omega U & -\omega B \\
O & (1-\alpha \tau) Q
\end{array}\right) \\
= & \left(\begin{array}{cc}
\frac{\tau}{1-\alpha \tau} Q^{-1} B^{T}(D-\omega L)^{-1}((1-\omega) D+\omega U) & I-\frac{\omega \tau}{1-\alpha \tau} Q^{-1} B^{T}(D-\omega L)^{-1} B
\end{array}\right), \\
& M(\omega, \tau, \alpha) \equiv \Omega^{-1}(D-\Omega L)=\left(\begin{array}{cc}
\frac{1}{\omega}(D-\omega L) & O \\
-B^{T} & \frac{1-\alpha \tau}{\tau} Q
\end{array}\right) .
\end{aligned}
$$

More precisely, we have the following algorithmic description of this GUSOR method.

Generalized USOR Method. Let $Q \in \mathbb{R}^{n \times n}$ be a prescribed symmetric positive definite matrix. Given initial vectors $x^{(0)} \in R^{m}$ and $y^{(0)} \in R^{n}$, and the relaxed parameters $\alpha, \omega$, and $\tau$ with $\omega, \tau \neq 0$. For $k=0,1,2, \ldots$ until the iteration sequence $\left\{\left(\left(x^{(k)}\right)^{T},\left(y^{(k)}\right)^{T}\right)^{T}\right\}$ converges, compute

$$
\begin{gathered}
x^{(k+1)}=(D-\omega L)^{-1} \\
\times\left(((1-\omega) D+\omega U) x^{(k)}\right. \\
\left.+\omega\left(b-B y^{(k)}\right)\right), \\
y^{(k+1)}=y^{(k)}+\frac{\tau}{1-\alpha \tau} Q^{-1}\left(B^{T} x^{(k+1)}-q\right) .
\end{gathered}
$$

Remark 1. When the relaxed parameters $\alpha=0$, the GUSOR method reduces to the USOR method, so the GUSOR method is the extention of the USOR method.

\section{Convergence of the GUSOR Method}

In this section, we will analyze a sufficient condition for parameters $\omega, \tau$, and $\alpha$ in the generalized Uzawa-SOR (GUSOR) method to solve augmented systems (2). We will use the following notations and definitions. For a vector $x$, $x^{*}$ denotes the complex conjugate transpose of the vector $x$. $\lambda_{\text {min }}(H)$ and $\lambda_{\text {max }}(H)$ denote the minimum and maximum eigenvalues of the Hermitian matrix $H$, respectively, and $\rho(H)$ denotes the spectral radius of $H$. We also assumed that the parameters of $\alpha, \omega$, and $\tau$ used in this paper are positive real numbers. 
Note that the iteration matrix of the proposed methods is $H(\omega, \tau, \alpha)$; therefore, the GUSOR method is convergent if and only if the spectral radius of the matrix $H(\omega, \tau, \alpha)$, defined in (8) is less than one; that is, $\rho(H(\omega, \tau, \alpha))<1$.

Let $\lambda$ be an eigenvalue of $H(\omega, \tau, \alpha)$ and let $\left(\begin{array}{l}x \\ y\end{array}\right)$ be the corresponding eigenvector. Then we have

$$
\begin{array}{r}
\left(\begin{array}{cc}
(1-\omega) D+\omega U & -\omega B \\
O & (1-\alpha \tau) Q
\end{array}\right)\left(\begin{array}{l}
x \\
y
\end{array}\right) \\
=\lambda\left(\begin{array}{cc}
D-\omega L & O \\
-\tau B^{T} & (1-\alpha \tau) Q
\end{array}\right)\left(\begin{array}{l}
x \\
y
\end{array}\right)
\end{array}
$$

or equivalently,

$$
\begin{gathered}
(1-\omega-\lambda) D x+\omega U x+\lambda \omega L x=\omega B y \\
(\lambda-1)(1-\alpha \tau) Q y=\lambda \tau B^{T} x .
\end{gathered}
$$

Lemma 2. Let $A$ be a symmetric positive definite matrix and $B$ a matrix of full column rank. If $\lambda$ is an eigenvalue of the iteration matrix $H(\omega, \tau, \alpha)$, then $\lambda \neq 1$.

Proof. If $\lambda=1$, since $\alpha$ and $\tau$ are positive real numbers, then from (12), we have $x=-A^{-1} B y$ and $B^{T} x=0$. It follows that $B^{T} A^{-1} B y=0$ from the above relations, which leads to $y=0$ and $x=0$. This is a contradiction to the assumption that $\left(\begin{array}{l}x \\ y\end{array}\right)$ is an eigenvector of the iteration matrix $H(\omega, \tau, \alpha)$.

Lemma 3. Let $A$ be a symmetric positive definite matrix, and $B$ a matrix of full column rank. If $\lambda$ is an eigenvalue of the iteration matrix $H(\omega, \tau, \alpha)$ and $\left(\begin{array}{l}x \\ y\end{array}\right)$ is an eigenvector of the iteration matrix $H(\omega, \tau, \alpha)$ corresponding to the eigenvalue $\lambda$, then $x \neq 0$. Moreover, if $y=0$, then $|\lambda|<1$.

Proof. The method of proof is exactly the same as in [12], here we omit the proof of Lemma 3.

Lemma 4 (see [13]). Both roots of the complex quadratic equation $\lambda^{2}-\phi \lambda+\psi=0$ have modulus less than one if and only if $|\phi-\bar{\phi} \psi|+|\psi|^{2}<1$, where $\bar{\phi}$ denotes the complex conjugate of $\phi$.

Now we are in the position to establish the convergence of the proposed methods. The following theorem presents a sufficient condition for guaranteeing the convergence of the GUSOR method.

Theorem 5. Let $A=D-L-U$ be a symmetric positive definite matrix, $B \in R^{m \times n}$ a matrix of full column rank, and let $Q \in$ $R^{n \times n}$ be a symmetric positive definite matrix. Let $S=i\left(L^{T}-\right.$ $L) / 2$. If $\alpha>0,0<\omega<2$ and $0<\tau<m_{0} /\left(1+\alpha m_{0}\right)$, where

$$
m_{0}=\frac{2(2-\omega) \min _{1 \leq j \leq n} a_{j j} \lambda_{\min }^{2}(A)}{\omega \rho\left(B Q^{-1} B^{T}\right)\left(4 \rho(S)^{2}+\lambda_{\min }^{2}(A)\right)}
$$

then the proposed method is convergent.
Proof. Since $\lambda \neq 1$ from Lemma 2, we have $\lambda \neq 1$. From (12), we obtain

$$
\begin{gathered}
y=\frac{\lambda \tau}{(\lambda-1)(1-\alpha \tau)} Q^{-1} B^{T} x, \\
(1-\omega-\lambda) D x+\omega U x+\lambda \omega L x \\
=\frac{\lambda \omega \tau}{(\lambda-1)(1-\alpha \tau)} B Q^{-1} B^{T} x .
\end{gathered}
$$

If $B^{T} x=0$, then $y=0$ and we have $|\lambda|<1$ from Lemma 3.

We now assume that $B^{T} x \neq 0$. For $x \neq 0$, let

$$
\begin{gathered}
\frac{x^{*} D x}{x^{*} x}=\delta, \quad \frac{x^{*} L^{T} x}{x^{*} x}=\alpha+\beta i, \\
\frac{x^{*} B Q^{-1} B^{T} x}{x^{*} x}=\gamma .
\end{gathered}
$$

Notice that $U^{T}=L$, then

$$
\begin{gathered}
\frac{x^{*} U^{T} x}{x^{*} x}=\alpha-\beta i \quad \delta>0, \gamma>0, \\
\delta-2 \alpha=\frac{x^{*} A x}{x^{*} x}>0 .
\end{gathered}
$$

Substituting into (15), we obtain

$$
\begin{gathered}
(1-\omega-\lambda) \delta+\omega(\alpha-\beta i)+\lambda \omega(\alpha+\beta i) \\
=\frac{\lambda \omega \tau}{(\lambda-1)(1-\alpha \tau)} \gamma .
\end{gathered}
$$

We notice $\delta-2 \alpha>0$ and $0<\omega<2$, that is, $-\delta+\omega \alpha+i \omega \beta \neq 0$, and after some manipulations, we get $\lambda$ satisfies the quadratic equation $\lambda^{2}-\phi \lambda+\psi=0$, where

$$
\begin{gathered}
\phi=\frac{(\omega-2) \delta+(\tau /(1-\alpha \tau)) \omega \gamma+2 \omega \beta i}{-\delta+\omega \alpha+i \omega \beta} \\
\varphi=\frac{(\omega-1) \delta+\omega \beta i-\omega \alpha}{-\delta+\omega \alpha+i \omega \beta}
\end{gathered}
$$

Let $\chi=(-\delta+\omega \alpha)^{2}+\omega^{2} \beta^{2}$. By some calculations, one has

$$
\begin{aligned}
|\bar{\phi} \varphi-\phi|= & \frac{1}{|1-\alpha \tau| \chi} \\
& \times\left(((1-\alpha \tau)(\omega-2) \delta+\tau \omega \gamma)^{2}\right. \\
& \left.\quad \times(\delta \omega-2 \omega \alpha)^{2}+\left(2 \tau \omega^{2} \beta \gamma\right)^{2}\right)^{1 / 2}, \\
|\varphi|^{2}= & \frac{1}{\chi}\left((\delta(\omega-1)-\omega \alpha)^{2}+\omega^{2} \beta^{2}\right) .
\end{aligned}
$$

From Lemma 4, we know that roots $\lambda$ of the complex quadratic equation (18) satisfy $|\lambda|<1$ if and only if

$$
|\phi-\bar{\phi} \varphi|+|\varphi|^{2}<1
$$


By solving (21) for $\tau$, if $0<\alpha \tau<1$, that is, $0<\tau<1 / \alpha$, one obtains

$$
0<\frac{\tau}{1-\alpha \tau}<\frac{2(\delta-2 \alpha)^{2}(2-\omega) \delta}{\left(4 \beta^{2}+(\delta-2 \alpha)^{2}\right) \omega \gamma} .
$$

Letting

$$
m=\frac{2(\delta-2 \alpha)^{2}(2-\omega) \delta}{\left(4 \beta^{2}+(\delta-2 \alpha)^{2}\right) \omega \gamma}
$$

we obtain

$$
0<\tau<\frac{m}{1+\alpha m}
$$

It is obviously that $0<\tau<1 / \alpha$. Since $S$ is a Hermitian matrix, the eigenvalues of $S$ are real, $0 \leq \beta^{2}=x^{*} S x / x^{*} x \leq \rho(S)^{2}$. For $\delta-2 \alpha=x^{*} A x / x^{*} x>0, \omega>0, \gamma>0$ and $z^{2} /\left(a+z^{2}\right)$ is an increasing function for $z>0$, we obtain that $|\lambda|<1$ when

$$
0<\tau<\frac{m_{0}}{1+\alpha m_{0}}
$$

where

$$
m_{0}=\frac{2(2-\omega) \min _{1 \leq j \leq n} a_{j j} \lambda_{\min }^{2}(A)}{\omega \rho\left(B Q^{-1} B^{T}\right)\left(4 \rho(S)^{2}+\lambda_{\min }^{2}(A)\right)}
$$

Hence, the theorem is proved.

\section{Numerical Experiments}

In this section, we provide numerical experiments to examine the feasibility and effectiveness of GUSOR method for solving the saddle point problem (1) and compare the results between the GUSOR method and the USOR method provided in [12]. We report the number of iterations (IT), norm of absolution residual vectors (RES), the elapsed CPU time (CPU), and the spectral radius of corresponding iterative matrix denoted by $\rho$. Here, RES is defined as

$$
\mathrm{RES}=\frac{\sqrt{\left\|b-A x^{(k)}-B^{T} y^{(k)}\right\|_{2}^{2}+\left\|q-B^{T} x^{(k)}\right\|_{2}^{2}}}{\sqrt{\|b\|_{2}^{2}+\|q\|_{2}^{2}}},
$$

with $\left(x_{k}^{T}, y_{k}^{T}\right)^{T}$ being the final approximate solution, where $\|\cdot\|$ refers to $L_{2}$-norm. We choose the right-hand vector $\left(b^{T}, q^{T}\right)^{T} \in R^{m+n}$ such that the exact solution of the augmented linear system (1) is $\left(x_{*}^{T}, y_{*}^{T}\right)^{T}=(1,1, \ldots, 1)^{T} \in$ $R^{m+n}$. All numerical experiments are carried out on a PC equipped with Intel Core i3 2.3 GHz CPU and 2.00 GB RAM memory Using MATLAB R2010a.
TABLE 1: Choices of the matrix $Q$.

\begin{tabular}{lcc}
\hline Case number & $Q$ & Description \\
\hline I & $\operatorname{tridiag}\left(B^{T} \widetilde{A}^{-1} B\right)$ & $\widetilde{A}=\operatorname{tridiag}(A)$ \\
II & $\operatorname{tridiag}\left(B^{T} A^{-1} B\right)$ & \\
III & $I$ & \\
\hline
\end{tabular}

Example 6 (see [9]). Let the augmented system (1) in which

$$
\begin{gathered}
A \&=\left(\begin{array}{cc}
I \otimes T+T \otimes I & O \\
O & I \otimes T+T \otimes I
\end{array}\right) \in R^{2 p^{2} \times 2 p^{2}}, \\
B=\left(\begin{array}{c}
I \otimes F \\
F \otimes I
\end{array}\right) \in R^{2 p^{2} \times 2 p^{2}}, \\
T=\frac{1}{h^{2}} \operatorname{tridiag}(-1,2,-1) \in R^{p \times p}, \\
F=\frac{1}{h} \operatorname{tridiag}(-1,1,0) \in R^{p \times p},
\end{gathered}
$$

with $\otimes$ is the Kronecker product symbol and $h=1 /(p+1)$ and $S=\operatorname{tridiag}(a, b, c)$ is a tridiagonal matrix with $S_{i, i}=b$, $S_{i-1, i}=a, S_{i, i+1}=c$ for appropriate $i$.

For this example, $m=2 p^{2}$ and $n=p^{2}$. Hence, the total number of variables is $m+n=3 p^{2}$. We choose the matrix $Q$ as an approximation to the matrix $B^{T} A^{-1} B$, according to three cases listed in Table 1.

In our experiments, all runs with respect to both USOR method and GUSOR method are started from the initial vector which is set to the zero vector and terminate if the current iteration satisfies ERR $<10^{-6}$. Here, ERR is defined as

$$
\mathrm{ERR}=\frac{\sqrt{\left\|x_{k}-x_{*}\right\|^{2}+\left\|y_{k}-y_{*}\right\|^{2}}}{\sqrt{\left\|x_{0}-x_{*}\right\|^{2}+\left\|y_{0}-y_{*}\right\|^{2}}} .
$$

In Tables 2,3 , and 4 , we list the values of $(\omega, \tau)$ which are same as in [12], IT, RES, CPU, and the spectral radii of corresponding iterative matrices for various problem sizes $(m$, $n$ ), respectively. They clearly show that the GUSOR method is more effective than the USOR method on convergence rate, computing speed, and the spectral radii of corresponding iterative matrices. IT and CPU of our proposed method are nearly half of the USOR if $p$ is smaller. However, the relaxed parameters $\alpha$ of GUSOR method are not optimal values and only lie in the convergence region of the method. The determination of optimum values of the parameters needs further study.

Remark 7. When $\alpha=0$, in this case, the proposed method is the one in [12]. Through experiment results, we find the optimal relaxation of $\alpha$ seems to be about 0.5 .

\section{Conclusions}

In this paper, we propose the GUSOR method for the solution of the saddle point problems and analyze the convergence 
TABLe 2: Numerical results for Example 6 in Case I with $\alpha=0.4$.

\begin{tabular}{lccc}
\hline$m$ & 128 & 512 & 1152 \\
$n$ & 64 & 256 & 576 \\
\hline USOR & & & \\
& 1.3 & 1.4 & 1.4 \\
$\tau$ & 0.6 & 0.5 & 0.5 \\
IT & 102 & 248 & 346 \\
RES & $3.18 e-8$ & $7.50 e-9$ & $1.80 e-8$ \\
CPU & 0.0073 & 0.5343 & 3.1231 \\
$\rho(H(\omega, \tau))$ & 0.8567 & 0.9387 & 0.9548 \\
\hline GUSOR & & & \\
$\omega$ & 1.3 & 1.4 & 1.4 \\
$\tau$ & 0.6 & 0.5 & 0.5 \\
IT & 63 & 179 & 282 \\
RES & $2.88 e-7$ & $1.52 e-9$ & $1.20 e-8$ \\
$\mathrm{CPU}$ & 0.0047 & 0.3611 & 2.5255 \\
$\rho(H(\omega, \tau, \alpha))$ & 0.7677 & 0.9146 & 0.9424 \\
\hline
\end{tabular}

TABLE 3: Numerical results for Example 6 in Case II with $\alpha=0.6$.

\begin{tabular}{lccc}
\hline$m$ & 128 & 512 & 1152 \\
$n$ & 64 & 256 & 576 \\
\hline USOR & & & \\
$\omega$ & 1.3 & 1.3 & 1.3 \\
$\tau$ & 0.5 & 0.5 & 0.5 \\
IT & 123 & 212 & 346 \\
RES & $2.53 e-8$ & $2.78 e-8$ & $1.75 e-8$ \\
CPU & 0.0169 & 0.4651 & 3.6313 \\
$\rho(H(\omega, \tau))$ & 0.8817 & 0.9263 & 0.9522 \\
\hline GUSOR & & & \\
$\omega$ & 1.3 & 1.3 & 1.3 \\
$\tau$ & 0.5 & 0.5 & 0.5 \\
IT & 62 & 169 & 280 \\
RES & $7.17 e-8$ & $2.16 e-8$ & $2.20 e-8$ \\
CPU & 0.0081 & 0.3728 & 2.9000 \\
$\rho(H(\omega, \tau, \alpha))$ & 0.7784 & 0.9027 & 0.9395 \\
\hline
\end{tabular}

of GUSOR method. When chosen the relaxed parameters $\alpha$, the spectral radii of the iteration matrices, IT and CPU with the proposed method are smaller than those in [12], which is shown through numerical experiments. Particularly, one may discuss how to select the set of optimal parameters for accelerating the convergence of the considered method effectively. The optimal choice of this set of parameters is valuably studied which is our future work.

\section{Acknowledgments}

The authors would like to thank the anonymous referees for their helpful comments and advice, which greatly improved the paper. The study was financially supported by the National Natural Science Foundation (nos. 11161041 and 71301111), Chinese Universities Specialized Research Fund for the Doctoral Program (20110185110020).
TABLE 4: Numerical results for Example 4.1 in Case III with $\alpha=0.4$.

\begin{tabular}{lccc}
\hline$m$ & 128 & 512 & 1152 \\
$n$ & 64 & 256 & 576 \\
\hline USOR & & & \\
$\omega$ & 1.3 & 1.4 & 1.3 \\
$\tau$ & 0.6 & 0.5 & 0.5 \\
IT & 120 & 265 & 364 \\
RES & $2.72 e-8$ & $7.40 e-9$ & $1.70 e-8$ \\
CPU & 0.0084 & 0.4487 & 3.2774 \\
$\rho(H(\omega, \tau))$ & 0.8783 & 0.9427 & 0.9549 \\
\hline GUSOR & & & \\
$\omega$ & 1.3 & 1.4 & 1.3 \\
$\tau$ & 0.6 & 0.5 & 0.5 \\
IT & 69 & 194 & 306 \\
RES & $5.02 e-8$ & $1.02 e-8$ & $1.98 e-8$ \\
CPU & 0.0054 & 0.3234 & 2.6347 \\
$\rho(H(\omega, \tau, \alpha))$ & 0.7890 & 0.9207 & 0.9448 \\
\hline
\end{tabular}

\section{References}

[1] S. Wright, "Stability of augmented system factorizations in interior-point methods," SIAM Journal on Matrix Analysis and Applications, vol. 18, no. 1, pp. 191-222, 1997.

[2] M. Arioli, I. S. Duff, and P. P. M. de Rijk, "On the augmented system approach to sparse least-squares problems," Numerische Mathematik, vol. 55, no. 6, pp. 667-684, 1989.

[3] J. Y. Yuan and A. N. Iusem, "Preconditioned conjugate gradient method for generalized least squares problems," Journal of Computational and Applied Mathematics, vol. 71, no. 2, pp. 287297, 1996.

[4] C. H. Santos, B. P. B. Silva, and J. Y. Yuan, "Block SOR methods for rank-deficient least-squares problems," Journal of Computational and Applied Mathematics, vol. 100, no. 1, pp. 1-9, 1998.

[5] J. Y. Yuan, "Numerical methods for generalized least squares problems," Journal of Computational and Applied Mathematics, vol. 66, no. 1-2, pp. 571-584, 1996.

[6] Z.-Z. Bai, "Structured preconditioners for nonsingular matrices of block two-by-two structures," Mathematics of Computation, vol. 75, no. 254, pp. 791-815, 2006.

[7] Z.-Z. Bai and G.-Q. Li, "Restrictively preconditioned conjugate gradient methods for systems of linear equations," SIMA Journal of Numerical Analysis, vol. 23, no. 4, pp. 561-580, 2003.

[8] G. H. Golub, X. Wu, and J.-Y. Yuan, "SOR-like methods for augmented systems," BIT Numerical Mathematics, vol. 41, no. 1, pp. 71-85, 2001.

[9] Z.-Z. Bai, B. N. Parlett, and Z.-Q. Wang, "On generalized successive overrelaxation methods for augmented linear systems," Numerische Mathematik, vol. 102, no. 1, pp. 1-38, 2005.

[10] M. T. Darvishi and P. Hessari, "Symmetric SOR method for augmented systems," Applied Mathematics and Computation, vol. 183, no. 1, pp. 409-415, 2006.

[11] G.-F. Zhang and Q.-H. Lu, "On generalized symmetric SOR method for augmented systems," Journal of Computational and Applied Mathematics, vol. 219, no. 1, pp. 51-58, 2008.

[12] J. Zhang and J. Shang, "A class of Uzawa-SOR methods for saddle point problems," Applied Mathematics and Computation, vol. 216, no. 7, pp. 2163-2168, 2010. 
[13] Z.-Z. Bai and Z.-Q. Wang, "On parameterized inexact Uzawa methods for generalized saddle point problems," Linear Algebra and Its Applications, vol. 428, no. 11-12, pp. 2900-2932, 2008.

[14] L. Wang and Z.-Z. Bai, "Skew-Hermitian triangular splitting iteration methods for non-Hermitian positive definite linear systems of strong skew-Hermitian parts," BIT Numerical Mathematics, vol. 44, no. 2, pp. 363-386, 2004. 


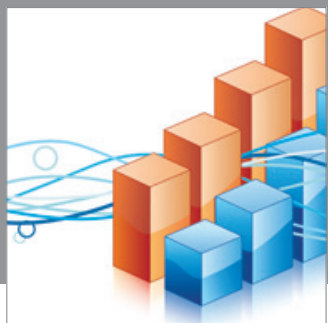

Advances in

Operations Research

mansans

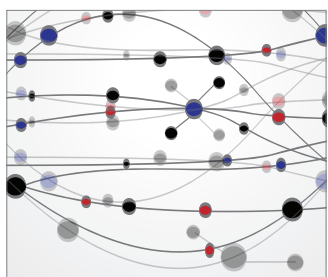

The Scientific World Journal
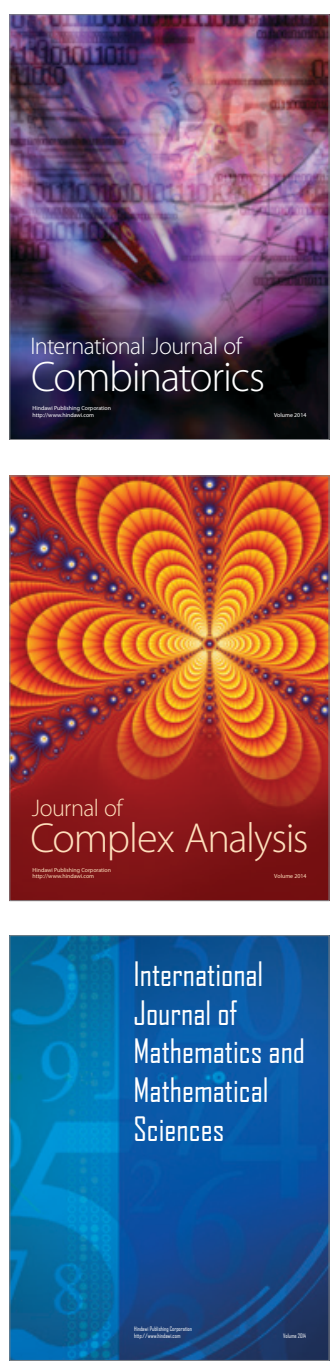
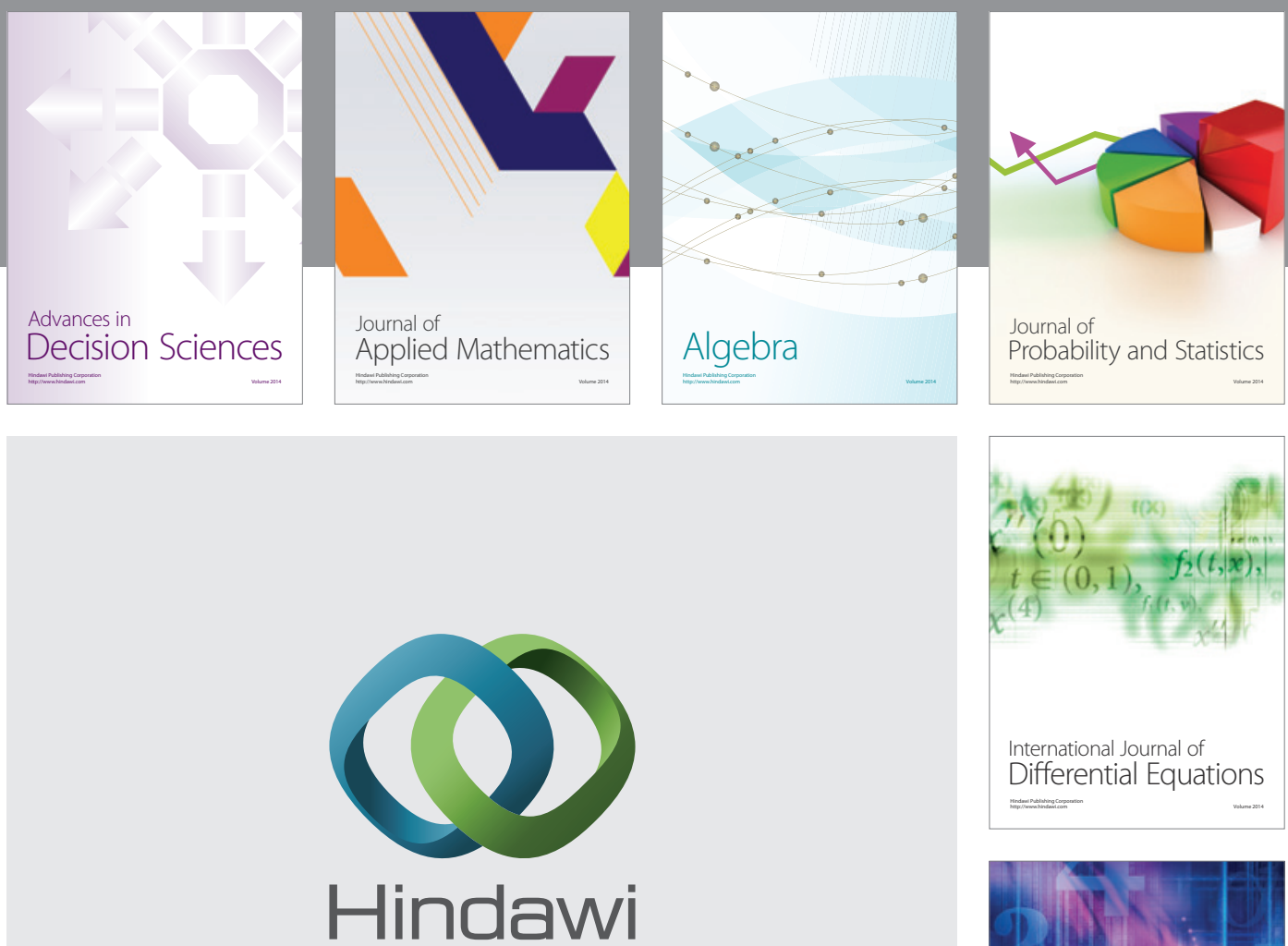

Submit your manuscripts at http://www.hindawi.com
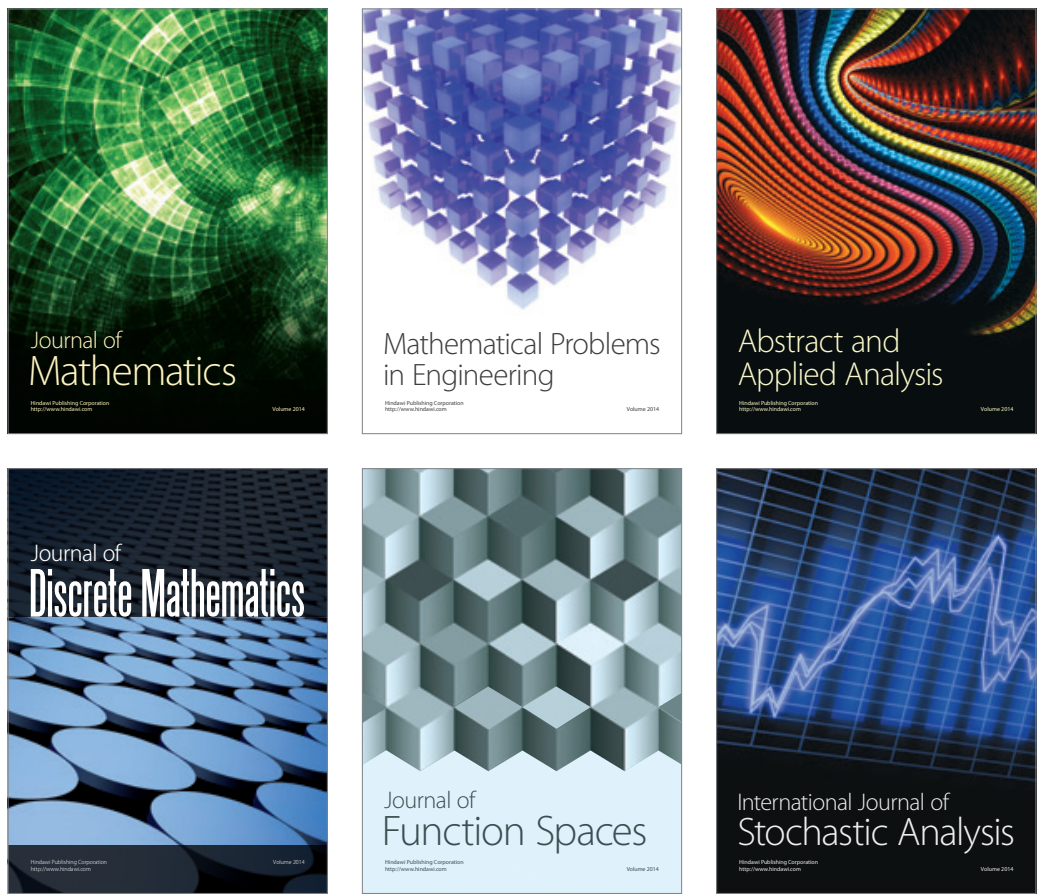

Journal of

Function Spaces

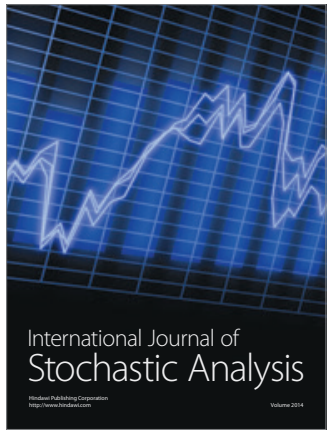

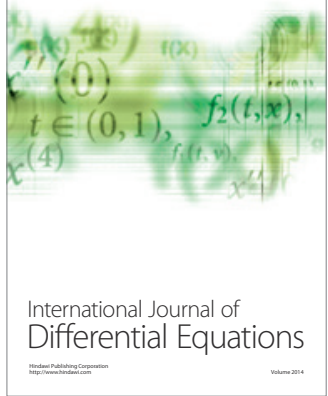
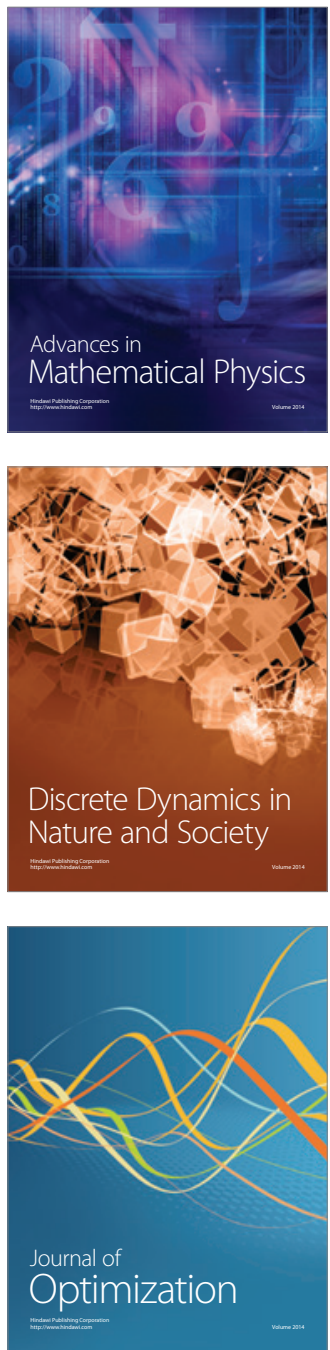